Artículo

\title{
Complejo PVA-quitosán-nCu mejora el rendimiento y la respuesta de defensa en tomate
}

\author{
Yoselin Athalia Rivera-Jaramillo ${ }^{1}$ \\ Gregorio Cadenas-Pliego ${ }^{2}$ \\ Adalberto Benavides-Mendoza ${ }^{3}$ \\ Alberto Sandoval-Rangel ${ }^{3}$ \\ Marcelino Cabrera-De la Fuente ${ }^{3}$ \\ Susana González-Morales ${ }^{4 \S}$ \\ ${ }^{1}$ Maestría en Ciencias en Horticultura-Universidad Autónoma Agraria Antonio Narro. Saltillo, México. CP. \\ 25315. (athalia_rivera@hotmail.com). ${ }^{2}$ Centro de Investigación en Química Aplicada. Saltillo, México. CP. \\ 25294. (gregorio.cadenas@ ciqa.edu.mx). ${ }^{3}$ Departameto de Horticultura-Universidad Autónoma Agraria \\ Antonio Narro. (abenmen@gmail.com; asandovalr16@gmail.com; cafum7@yahoo.com). ${ }^{4}$ CONACYT- \\ Universidad Autónoma Agraria Antonio Narro. \\ ${ }^{\S}$ Autora para correspondencia: sgonzalezmo@conacyt.mx.
}

\section{Resumen}

Actualmente el uso de la nanotecnología está revolucionando la producción agrícola. Se ha demostrado que las nanopartículas de cobre tienen un efecto en el crecimiento y desarrollo de las diferentes especies vegetales, además de operar como inductores de resistencia al estrés. El objetivo del presente trabajo fue evaluar la respuesta en el crecimiento y rendimiento, así como la activación del sistema de defensa de plantas de tomate. Los tratamientos evaluados fueron un complejo de alcohol polivinílico-quitosán-nanopartículas de cobre (PVA-Cts-nCu), otro complejo de PVA-Cts y un testigo absoluto (T0). Los tratamientos se aplicaron vía foliar en plantas de tomate bajo condiciones de invernadero. Durante el ciclo del cultivo, se determinaron variables agronómicas, y la actividad de enzimas relacionadas a la tolerancia a estrés como $\beta-1,3$ glucanasa, quitinasa y fenilalanina amonio liasa (PAL), así como la expresión del gen PR1. El complejo PVA-Cts-nCu incrementó el rendimiento, número de frutos, peso promedio de fruto, peso fresco aéreo y peso fresco de la raíz, además, promovió el sistema de defensa mediante el aumento en la actividad enzimática PAL, así como la sobreexpresión del gen PR1.

Palabras clave: bioestimulante, estrés, expresión de genes, hortalizas.

Recibido: junio de 2021

Aceptado: agosto de 2021 


\section{Introducción}

El sector agrícola enfrenta actualmente desafíos importantes de aumentar la productividad para alimentar a la población mundial en crecimiento e incrementar la eficiencia en el uso de los recursos (Rouphael y Colla, 2020). Lo anterior, ha motivado a ampliar el campo de la investigación de la nanotecnología y sus posibles aplicaciones en el campo agrícola (Usman et al., 2020). Las nanopartículas (NPs) tienen una superficie altamente reactiva, por lo tanto, son biológicamente activas, esto debido a que su área superficial es extremadamente alta proporcional a su volumen (Pestovsky y Martínez, 2017). Se sintetizan en diferentes tamaños, formas, materiales y reactividad, esto les otorga la capacidad de potenciar el sector agrícola (Fatima et al., 2021).

El cobre $(\mathrm{Cu})$ es un micronutriente ampliamente distribuido en los tejidos vegetales, participa en procesos fisiológicos y es esencial para el crecimiento de las plantas (Rajput et al., 2018). Se ha demostrado que activa enzimas claves en el ciclo de Calvin, mejorando la actividad fotosintética e incrementando el rendimiento en las plantas (Pradhan et al., 2015). Las NPs de $\mathrm{Cu}$ pueden funcionar como reductores u oxidantes en reacciones bioquímicas dentro de la célula por lo que pueden catalizar la producción de especies reactivas de oxígeno (ROS) e inducir estrés oxidativo (Somasundaran et al., 2010). Los efectos de las NPs de Cu varían, se ha reportado que a bajas concentraciones incrementa la tasa de crecimiento y la germinación de varias plantas, por otro lado, a concentraciones altas el crecimiento es retardado (Kasana $e t$ al., 2017). Además, en aplicaciones foliares con NPs de $\mathrm{Cu}$, favorecen la firmeza de frutos y contenido de antioxidantes como vitamina C, licopeno y fenoles (López-Vargas et al., 2018; Pérez-Labrada et al., 2019).

El quitosán (Cts) se ha utilizado para mejorar las defensas naturales de las plantas, entre las respuestas de defensa se incluyen la lignificación, activación de enzimas asociadas a la respuesta a patógenos en plantas, biosíntesis de fitoalexinas, generación de ROS, biosíntesis del ácido jasmónico, salicílico, abscísico y la expresión de genes relacionados con la defensa (El Hadrami et al., 2010). La aplicación del quitosán incrementa la actividad de $\beta-1,3$ glucanasa y quitinasa en diferentes especies vegetales, ayudando a hidrolizar la pared celular de hongos fitopatógenos (Rodríguez-Pedroso et al., 2009; González Peña et al., 2014).

El alcohol polivinílico (PVA) se ha utilizado ampliamente en la preparación de hidrogeles y en la inhibición del crecimiento bacteriano, además, tiene un alto potencial como un sistema de liberación controlada (Yang et al., 2016). La mezcla de PVA-Cts ha demostrado tener efectos in vitro sobre la respuesta antioxidante eliminando radicales libres, así como sobre la viabilidad de Staphylococcus aureus 8325-4 y Escherichia coli RB (Yang et al., 2018).

Actualmente existen pocos estudios sobre la aplicación de complejos de PVA-Cts-nCu. Estos complejos aplicados en forma de hidrogeles incrementan la calidad nutracéutica en frutos de tomate (Hernández-Hernández et al., 2018a). También promueven el incremento del vigor y número de racimos florales en tomate (Hernández-Hernández et al., 2017). Aplicados en sustrato incrementan el rendimiento en frutos de chile jalapeño (Pinedo-Guerrero et al., 2017). El objetivo del presente trabajo fue evaluar la respuesta del complejo PVA-Cts-nCu en el crecimiento y calidad de fruto, así como la activación del sistema de defensa de plantas de tomate. 


\section{Materiales y métodos}

\section{Síntesis del complejo PVA-Cts y PVA-Cts-nCu}

Para la preparación del complejo PVA-Cts-nCu, 1.5 L de PVA (BP-05, Nacional PIM México SA de CV, peso molecular de $\left.27000-32000 \mathrm{~g} \mathrm{~mol}^{-1}\right)$ al $1 \%(\mathrm{p} / \mathrm{v})$ se mezclaron con $1.5 \mathrm{~L}$ de Cts (Marine Chemicals, India, peso molecular de $\left.200000 \mathrm{~g} \mathrm{~mol}^{-1}\right)$ al $0.5 \%(\mathrm{p} / \mathrm{v})$. El nCu (1.85 g) se molió con $3 \mathrm{ml}$ de Agrex $^{\circledR} \mathrm{F}$ (Agroenzymas ${ }^{\circledR}$ SA de CV) como dispersante para las NPs. El nCu presentó una morfología esférica y semiesférica y un tamaño promedio de $30 \mathrm{~nm}$ (Sierra-Ávila $e t$ al., 2014, 2015).

\section{Establecimiento del cultivo}

La prueba se llevó a cabo en un invernadero de tipo túnel. Se usó un sistema de cultivo sin suelo,

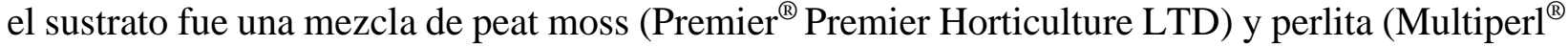
Grupo perlita de la laguna) (1:1 v/v). Se utilizaron plantas de tomate hibrido tipo saladett de crecimiento indeterminado Cid F1 (Harris Moran ${ }^{\circledR}$ ), se colocaron en bolsas de polietileno de $14 \mathrm{~L}$.

El experimento se llevó a cabo aplicando los tratamientos PVA-Cts-nCu $500 \mathrm{mg} \mathrm{L}^{-1}$, PVA-Cts 500 $\mathrm{mg} \mathrm{L}^{-1} \mathrm{y}$ un testigo absoluto con agua destilada (T0). La aplicación de los tratamientos fue foliar, con un gasto de $75 \mathrm{ml}$ de solución por planta durante el ciclo productivo. Las plántulas tuvieron una aplicación dos días previos al trasplante, posterior se aplicó en intervalos de 20 días iniciando después del trasplante. Se usó como medio de nutrición la solución Steiner (Steiner 1961).

\section{Variables de desarrollo y productividad en tomate}

La evaluación de la altura de planta y diámetro de tallo se llevó a cabo a los 120 días después del trasplante (DDT). A los 120 DDT se determinó el rendimiento por planta. Además, se determinó el peso fresco aéreo, así como el peso fresco de la raíz.

\section{Extracción de enzimas}

La actividad de las enzimas fenilalanina amonio liasa (PAL), quitinasa y $\beta$-1,3 glucanasa se analizó en hojas en tres muestreos distintos, al momento del trasplante ( $48 \mathrm{~h}$ posteriores a la primera aplicación del complejo), a los 40 DDT y 80 DDT. Las hojas fueron colectadas con nitrógeno líquido.

La extracción de biomoléculas se realizó de acuerdo con Rodríguez-Pedroso et al. (2006). La actividad PAL se determinó de acuerdo con Sykłowska-Baranek et al. (2012). Los resultados se expresaron como $\mathrm{U}$ (producción de $\mu \mathrm{mol}$ equivalente de ácido transcinámico por mililitro por minuto) por proteínas totales $\left(\mathrm{mg} \mathrm{g}^{-1}\right)$. La actividad de quitinasa se determinó de acuerdo con Rodríguez-Pedroso et al. (2006). Los resultados se expresaron como U (producción de $\mu \mathrm{g} \mathrm{ml}^{-1}$ de glucosa por minuto por proteínas totales $\left(\mathrm{mg} \mathrm{g}^{-1}\right)$. La actividad $\beta-1,3$ glucanasa se determinó de acuerdo con Rodríguez-Pedroso et al. (2006). La actividad enzimática se determinó por la medición del nivel de producción de azúcares reductores y se expresó en términos de producción de $\mu \mathrm{g} \mathrm{ml}^{-1}$ de glucosa por minuto por proteínas totales $\left(\mathrm{mg} \mathrm{g}^{-1}\right)$. 


\section{Expresión del gen PR1}

Las muestras de hojas se colectaron en tres tiempos diferentes, iniciando a las $48 \mathrm{~h}$ posteriores a la primera aplicación del complejo, después de este primer muestreo los siguientes fueron cada 40 días. Se colectaron hojas jóvenes completamente expandidas con nitrógeno líquido. La extracción de ARN se llevó a cabo con la técnica de $\mathrm{TRIzol}^{\mathrm{TM}}$ Reagent (Invitrogen).

El cDNA se sintetizó con el kit SensiFAST ${ }^{\mathrm{TM}}$ (Bioline). Los primers utilizados corresponden a un gen endógeno (Actina) y al gen PR1. ACT (fwd 5'-CCCAGGCACACAGGTGTTAT-3'; rev 5'CAGGAGCAACTCGAAGCTCA-3'); PR1 (fwd 5'-AAGTAGTCTGGCGCAACTCA-3'; rev 5'GTCCGATCCAGTTGCCTACA-3'). La cuantificación del gen PR1 se llevó a cabo en un equipo de PCR en tiempo real (Applied Biosystems StepOne ${ }^{\mathrm{TM}}$ versión 2.3) por el método $\Delta \Delta \mathrm{Ct}$ midiendo la intensidad de fluorescencia del Sybr Green (Hernández-Hernández et al., 2018b).

\section{Análisis de datos}

En la prueba en plantas de tomate se usó un diseño completamente al azar. Para las variables agronómicas se analizaron 20 unidades experimentales por cada tratamiento. En la cuantificación de la actividad PAL, quitinasa y $\beta-1,3$ glucanasa se analizaron cinco repeticiones por tratamiento y en la expresión del gen PR1 se analizaron cuatro repeticiones compuestas (10 plantas por repetición). Para detectar diferencias estadísticas entre tratamientos se realizó un análisis de varianza (Anova) y se efectuó una prueba de separación de medias según la prueba LSD de Fisher $(p \leq 0.05)$. Todos los análisis estadísticos se realizaron en el software estadístico InfoStat v2018.

\section{Resultados y discusión}

\section{Efecto del complejo PVA-Cts-nCu sobre variables de desarrollo y productividad en tomate}

La aplicación foliar del complejo PVA-Cts-nCu tuvo efectos significativos $(p \leq 0.5)$ en las variables relacionadas al vigor en las plantas de tomate (Cuadro 1). El tratamiento PVA-Cts-nCu presentó diferencias significativas en comparación con el testigo, incrementando el rendimiento (60.68\%), el peso promedio de fruto (18.2\%), el número de frutos (35.99\%), el peso fresco aéreo (26.99\%) y el peso fresco de la raíz (80.87\%). Mientras que el tratamiento PVA-Cts aumentó el peso promedio de fruto $(8.39 \%)$ y el peso fresco de la raíz (52.22\%) en comparación al testigo. En la variable de altura y diámetro de tallo no se observaron diferencias significativas.

Cuadro 1. Efecto del complejo PVA-Cts-nCu y PVA-Cts sobre el crecimiento y productividad del tomate.

\begin{tabular}{cccccccc}
\hline Tratamiento & $\begin{array}{c}\text { Altura } \\
(\mathrm{cm})\end{array}$ & $\begin{array}{c}\text { DT } \\
(\mathrm{mm})\end{array}$ & $\begin{array}{c}\text { Rendimiento } \\
\left(\mathrm{g} \mathrm{planta}^{-1}\right)\end{array}$ & $\begin{array}{c}\text { PPF } \\
\left(\mathrm{g} \mathrm{fruto}^{-1}\right)\end{array}$ & $\mathrm{NF}$ & $\begin{array}{c}\text { PFA } \\
\left(\mathrm{g} \mathrm{planta}^{-1}\right)\end{array}$ & $\begin{array}{c}\text { PFR } \\
\left(\mathrm{g} \mathrm{planta}^{-1}\right)\end{array}$ \\
\hline T0 & $132.29 \mathrm{a}$ & $17.02 \mathrm{a}$ & $2573.31 \mathrm{~b}$ & $58.62 \mathrm{c}$ & $43.9 \mathrm{~b}$ & $2384 \mathrm{~b}$ & $98.8 \mathrm{~b}$ \\
PVA-Cts-nCu & $134.78 \mathrm{a}$ & $16.79 \mathrm{a}$ & $4134.95 \mathrm{a}$ & $69.29 \mathrm{a}$ & $59.7 \mathrm{a}$ & $3027.65 \mathrm{a}$ & $175.8 \mathrm{a}$ \\
PVA-Cts & $119.71 \mathrm{a}$ & $15.04 \mathrm{a}$ & $2690.92 \mathrm{~b}$ & $63.54 \mathrm{~b}$ & $42.35 \mathrm{c}$ & $2299.47 \mathrm{~b}$ & $150.4 \mathrm{a}$ \\
\hline
\end{tabular}

$\mathrm{T} 0=$ testigo; $\mathrm{DT}=$ diámetro de tallo; $\mathrm{NF}=$ número de frutos; $\mathrm{PPF}=$ peso promedio de fruto; $\mathrm{NF}=$ número de frutos; $\mathrm{PFA}=$ peso fresco aéreo; $\mathrm{PFR}=$ peso fresco de la raíz, medias con la misma letra por columna son estadísticamente iguales (LSD Fisher, $p \leq 0.05$ ). 
Los resultados obtenidos en este estudio demuestran que los complejos PVA-Cts-nCu y PVA-Cts aplicados vía foliar no presentan toxicidad en las plantas de tomate. Rajput et al. (2018) mencionan que la fitotoxicidad dependerá de la concentración, tipo y tamaño de las NPs, además de la especie en donde son aplicadas, las condiciones de crecimiento y el tiempo de exposición.

Saharan et al. (2015) reportan un incremento en la biomasa en tomate tratadas con NPs de CuCts. Adhikari et al. (2012) observaron que la aplicación de NPs de CuO incrementó el crecimiento de la raíz en soya y garbanzo. En el presente estudio demostró que el complejo PVA-Cts-nCu mejoró el crecimiento aumentando el peso fresco aéreo y de raíz, parámetros importantes para el desarrollo del cultivo. Este efecto pudo ser inducido por la sinergia entre el nCu y el quitosán. Ya que este biopolímero y el $\mathrm{nCu}$ han demostrado tener efecto sobre el crecimiento y desarrollo de las plantas (El Hadrami et al., 2010; Hernández et al., 2017; Pinedo-Guerrero et al., 2017).

En la etapa de producción, el complejo PVA-Cts-nCu aumentó el rendimiento, el número de frutos y el peso promedio de fruto. Esto coincide con lo reportado por Pinedo-Guerrero et al. (2017), quienes señalan que al aplicar el hidrogel de PVA-Cts-nCu directo al suelo en chile jalapeño, incrementó el rendimiento $(8.27 \%)$ y el número de frutos $(9.32 \%)$ por planta. También HernándezHernández et al. (2018a) reportaron que la aplicación de hidrogeles de PVA-Cts-nCu directo al sustrato se incrementó en plantas de tomate el número de frutos $(20 \%)$.

Pradhan et al. (2015) evaluaron la aplicación de NPs de Cu en soja verde, donde las NPs influyeron en la actividad de enzimas claves en el ciclo de Calvin (fructosa-1, 6-bifosfato fosfatasa, ribulosa5-fosfato quinasa y NADP-gliceraldehído-3-fosfato deshidrogenasa). Es probable que al incrementarse la actividad de enzimas relacionadas con el transporte de electrones en la fotosíntesis se modificara la tasa de producción de fotosintatos y la velocidad a la cual se movilizan, incrementándose así la producción de frutos y el peso de estos.

\section{Efecto del complejo PVA-Cts-nCu sobre la actividad PAL}

La aplicación foliar de PVA-Cts-nCu tuvo efectos significativos $(p \leq 0.5)$ en la actividad PAL en hojas (Figura 1). El tratamiento PVA-Cts-nCu exhibe un incremento en la actividad PAL al trasplante (301.7\%) y a los 80 DDT (55.04\%) en comparación al testigo. A los 40 DDT el tratamiento PVA-Cts-nCu incrementó la actividad PAL en un $369.23 \%$ en comparación con PVA-Cts.

El quitosán y las NPs son conocidos como inductores potenciales para mejorar la defensa en las plantas ante el estrés, dando como resultado una acumulación de metabolitos relacionados con la defensa (Zhang y Liu, 2015; Usman et al., 2020), así como la generación de ROS y el incremento de la actividad de proteínas relacionadas con la defensa (González-Peña et al., 2014).

La enzima PAL es clave en la síntesis de fenoles y flavonoides, los fenoles son importantes precursores de compuestos que intervienen en el control del crecimiento vegetal, además de ser potentes antioxidantes (Santos-Sanchez et al., 2019).

El incremento de PAL puede derivarse de la interacción directa de las $\mathrm{nCu}$ y el Cts generando un efecto sinérgico. Cumplido-Nájera et al. (2019) aplicaron NPs de $\mathrm{Cu}$ de manera foliar incrementando 1.78 veces la actividad de PAL en hojas. 


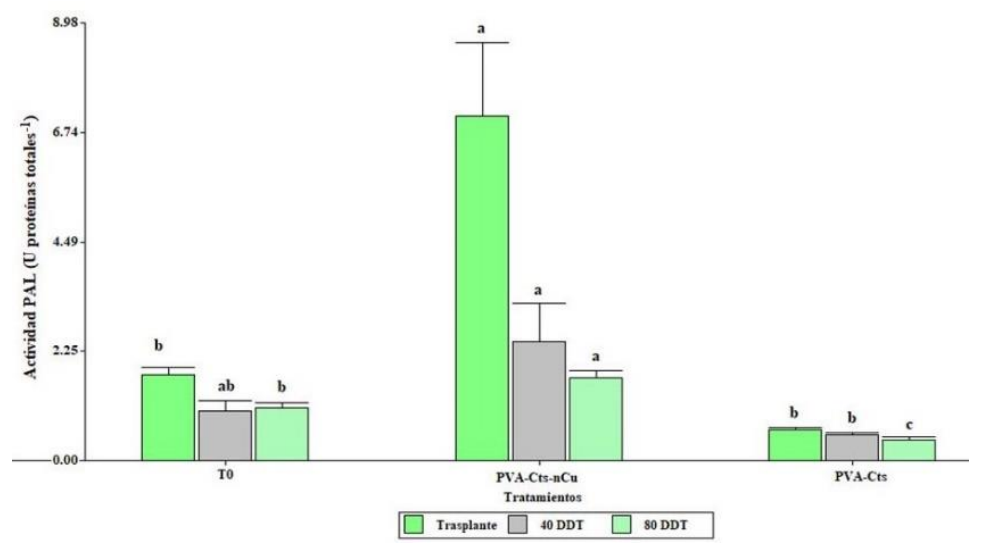

Figura 1. Actividad enzimática de fenilalanina amonio liasa en tomate con aplicación de PVA-Cts-nCu y PVA-Cts. Medias con la misma letra son estadísticamente iguales LSD Fisher, $p \leq 0.05$.

Falcón Rodríguez et al. (2012) reportaron que al asperjar quitosán $\left(0.5,1\right.$ y $\left.2.5 \mathrm{~g} \mathrm{~L}^{-1}\right)$ en plantas de tabaco todas incrementaron la actividad de PAL en hoja. Rodríguez et al. (2006), encontraron que al tratar previamente semillas de arroz con hidrolizados de quitosán $\left(500 \mathrm{mg} \mathrm{L}^{-1}\right)$ se estimuló la actividad de PAL en hoja. En un ensayo de Hernández-Hernández et al. (2018a) con plantas de tomate bajo estrés salino encontraron que la aplicación PVA-Cts-nCu sin estrés salino y PVA-Cts bajo estrés salino incrementaba la actividad de PAL en hoja. González-Peña et al. (2014) reportan que al asperjar quitosán $\left(100 \mathrm{mg} \mathrm{L}^{-1}\right)$ en plántulas de tomate hubo incremento de la actividad PAL. Chandra et al. (2015) aplicaron de manera foliar NPs de Cts en Camellia sinensis L. encontrando un aumento significativo en la acumulación de peroxidasa, polifenol oxidasa y PAL en hoja.

\section{Efecto del complejo PVA-Cts-nCu sobre la actividad $\beta-1,3$ glucanasa y quitinasa}

La aplicación foliar de ambos tratamientos no tuvo efectos significativos $(p \leq 0.5)$ con respecto al testigo; sin embargo, el PVA-Cts-nCu mostró un incremento de la actividad $\beta-1,3$ glucanasa respecto a PVA-Cts (162.85\%) a los 40 DDT. A los 80 DDT el T0 incrementó la actividad por encima de PVA-Cts-nCu y PVA-Cts (Figura 2).

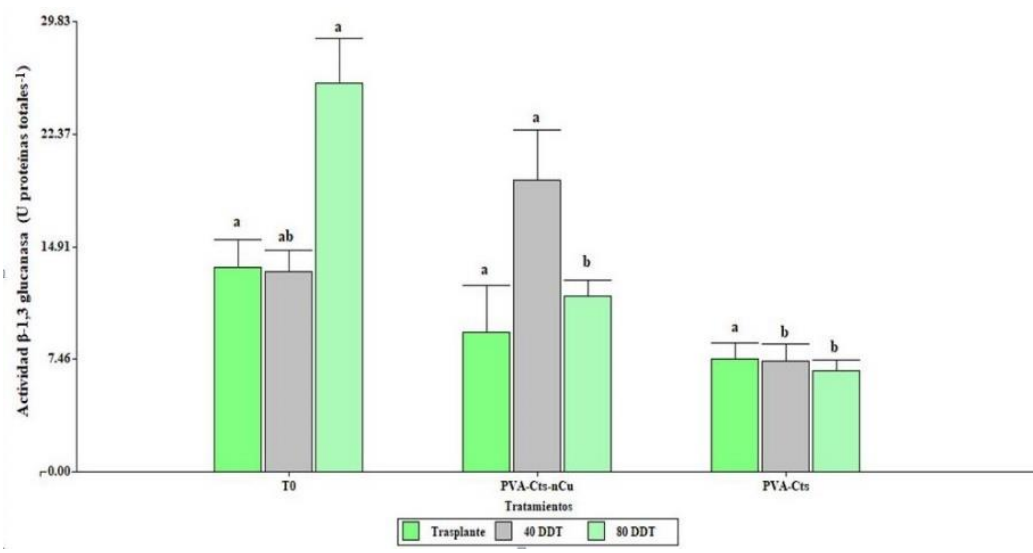

Figura 2. Actividad enzimática de $\beta-1,3$ glucanasa en plantas de tomate con la aplicación de PVA-CtsnCu y PVA-Cts. Medias con la misma letra son estadísticamente iguales LSD Fisher, $p \leq 0.05$. 
La aplicación foliar de PVA-Cts-nCu tuvo efectos significativos $(p \leq 0.5)$ en la actividad de quitinasa (Figura 3). A los 40 DDT el tratamiento PVA-Cts-nCu incrementó la actividad en $121 \%$ en comparación con PVA-Cts. A los 80 DDT, T0 incrementó la actividad por encima de PVA-Cts$\mathrm{nCu}$ y PVA-Cts. El tratamiento PVA-Cts-nCu incrementó la actividad en $54.67 \%$ en comparación con PVA-Cts.

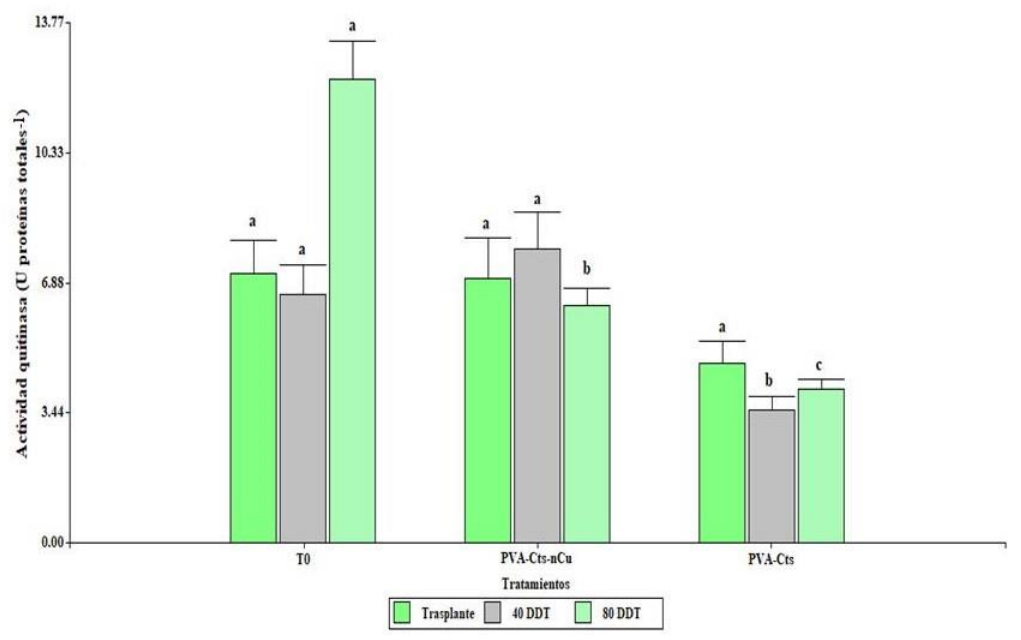

Figura 3. Actividad enzimática de quitinasa en plantas de tomate con la aplicación de PVA-Cts-nCu y PVA-Cts. Medias con la misma letra son estadísticamente iguales LSD Fisher, $p \leq 0.05$.

Las proteínas relacionadas a la patogenicidad (PR) como la $\beta-1,3$ glucanasa y quitinasa pueden ser expresadas de forma constitutiva e inducida en respuesta a una infección. Con respecto a la eficacia del quitosán en la inducción de la defensa en la planta, en hojas de Camellia sinensis L., aumentó la actividad de $\beta$-1,3-glucanasa $29.26 \%$, así como el contenido de peroxidasa y polifenol oxidasa (Chandra et al., 2015). Al aplicar NPs Cts y Cts en plantas de tomate estas incrementaron la expresión relativa de los genes relacionados con la síntesis de quitinasa y $\beta-1,3$ glucanasa en comparación al testigo (Chun y Chandrasekaran, 2019).

\section{Efecto de PVA-Cts-nCu en la expresión del gen PR1}

Los resultados de la expresión del gen PR1 se presentan en la Figura 4. El tratamiento PVACts-nCu sobreexpresó el gen PR1 (268.3, 0.72 y 8 veces respecto al T0) al trasplante, 40 y 80 DDT. Al igual que PVA-Cts-nCu, el tratamiento PVA-Cts sobreexpresó el gen PR1 (3.9, $1.5 \mathrm{y}$ 123.2 veces respecto a T0) al trasplante, 40 y 80 DDT. Estos resultados sugieren que el complejo PVA-Cts-nCu podría jugar un papel importante en la activación de genes que codifican a la producción de proteínas PR, relacionadas con la vía de señalización del ácido salicílico y la resistencia sistémica adquirida (RSA) ligada a la tolerancia al estrés (AbuQamar et al., 2009).

Existen evidencias de que el quitosán puede activar la respuesta de defensa en las plantas, incrementando la actividad de enzima PAL que está directamente relacionada con la síntesis de ácido salicílico, induciendo así la síntesis de proteínas PR, involucradas en los mecanismos de la RSA (Rodríguez-Pedroso et al., 2006; Sánchez et al., 2010; González Peña et al., 2014). 


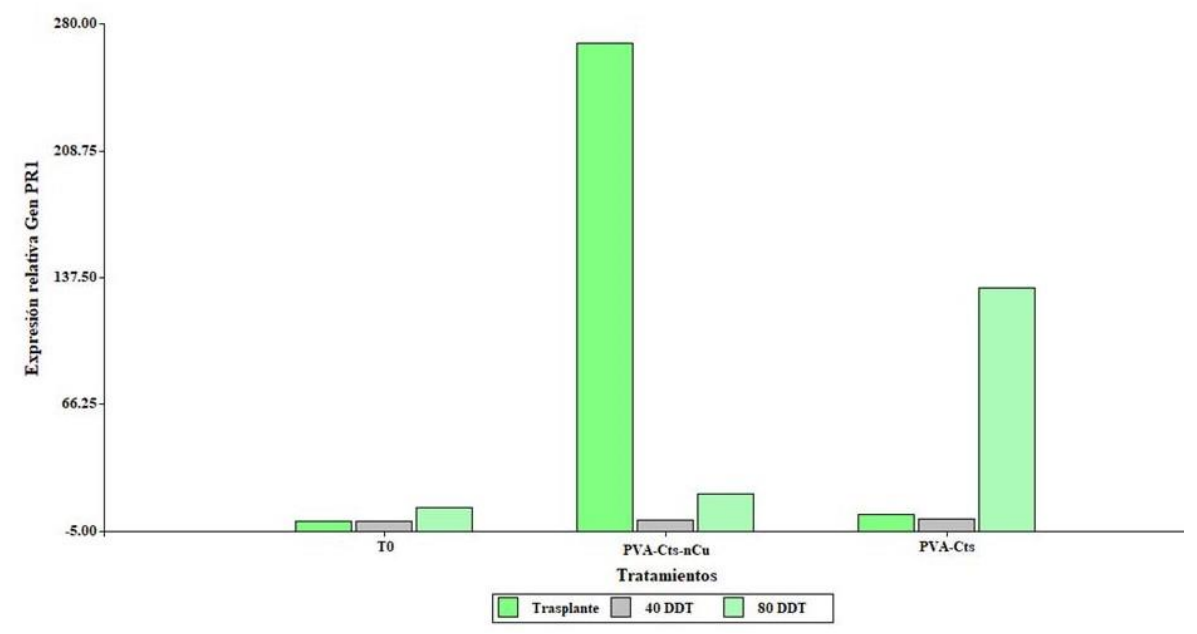

Figura 4. Expresión relativa del gen PR1 en hojas de plantas de tomate tratadas con PVA-Cts-nCu y PVA-Cts.

Chun y Chandrasekaran (2019) aplicaron NPs de Cts de manera foliar en plantas de tomate inoculadas con Fusarium andiyazi, estas incrementaron la expresión relativa del gen PR1 en hoja en comparación con el testigo. Hernández-Hernández et al. (2018b) aplicaron hidrogeles de PVA$\mathrm{Cts}-\mathrm{nCu}$ directo al sustrato en tomate bajo condiciones de estrés salino y encontraron que se reprimía la expresión del gen PR1 bajo condición salina y sin estrés salino, sin embargo, se sobreexpresaba el gen JA relacionado con la vía de los jasmonatos y esta mediada por la resistencia sistémica inducida (RSI).

Estos resultados arrojan que los complejos PVA-Cts-nCu y PVA-Cts pueden ser inductores potenciales para mediar la expresión de genes PR1 y JA en plantas de tomate activando la cascada de señalización de síntesis de compuesto fenólicos y enzimas que prepararan a la planta contra los efectos del estrés, ya sea por la vía del ácido salicílico (RSA) o la vía del ácido jasmónico (RSI).

\section{Conclusiones}

Este estudio demostró que el alcohol polivinílico-quitosán-nanocobre y el alcohol polivinílicoquitosán incrementaron las variables relacionadas con el vigor en las plantas, el rendimiento, además de activar el mecanismo de defensa de las plantas mediante el aumento de enzimas como PAL, $\beta-1,3$ glucanasa y quitinasa, además de incrementar la expresión del gen PR1 ligados con la tolerancia al estrés en plantas.

\section{Agradecimientos}

Este trabajo fue realizado gracias al Consejo Nacional de Ciencia y Tecnología (CONACYT) número de beca 887137. Maestría en Ciencias en Horticultura-Universidad Autónoma Agraria Antonio Narro. Saltillo, México. G. C. P. por la contribución de reactivos y materiales. Centro de Investigación en Química Aplicada. Saltillo, México. 


\section{Literatura citada}

AbuQamar, S.; Luo, H.; Laluk, K.; Mickelbart, M. V. and Mengiste, T. 2009. Crosstalk between biotic and abiotic stress responses in tomato is mediated by the AIM1 transcription factor. The Plant J. 58(2):347-360.

Adhikari, T.; Kundu, S.; Biswas, A. K.; Tarafdar, J. C. and Rao, A. S. 2012. Effect of copper oxide nano particle on seed germination of selected crops. J. Agric. Sci. Technol. 2(6A):815-823.

Chandra, S.; Chakraborty, N.; Dasgupta, A.; Sarkar, J.; Panda, K. and Acharya, K. 2015. Chitosan nanoparticles: a positive modulator of innate immune responses in plants. Scientific reports. $5(1): 1-14$.

Chun, S. C. and Chandrasekaran, M. 2019. Chitosan and chitosan nanoparticles induced expression of pathogenesis-related proteins genes enhances biotic stress tolerance in tomato. Inter. J. Biol. Macromol. 125(51):948-954.

Cumplido-Nájera, C. F.; González-Morales, S.; Ortega-Ortíz, H.; Cadenas-Pliego, G.; BenavidesMendoza, A. and Juárez-Maldonado, A. 2019. The application of copper nanoparticles and potassium silicate stimulate the tolerance to Clavibacter michiganensis in tomato plants. Sci. Hortic. 245(1):82-89.

El Hadrami, A.; Adam, L. R.; El Hadrami, I. and Daayf, F. 2010. Chitosan in plant protection. Marine drugs. 8(4):968-987.

Falcón-Rodríguez, A.; Costales-Menéndez, D.; Martínez-Téllez, M. Á. y Gordon, T. A. 2012. Respuesta enzimática y de crecimiento en una variedad comercial de tabaco (Nicotiana tabacum, L.) tratada por aspersión foliar de un polímero de quitosana. Cultivos Tropicales. 33(1):65-70.

Fatima, F.; Hashim, A. and Anees, S. 2021. Efficacy of nanoparticles as nanofertilizer production: a review. Environ. Sci. Pollut. Res. 28(2):1292-1303.

Fish, W. W.; Perkins-Veazie, P. and Collins, J. K. 2002. A quantitative assay for lycopene that utilizes reduced volumes of organic solvents. J. Food Composition and Analysis. 15(3):309-317.

González-Peña, D.; Costales, D. y Falcón, A. B. 2014. Influencia de un polímero de quitosana en el crecimiento y la actividad de enzimas defensivas en tomate (Solanum lycopersicum L.). Cultivos Tropicales. 35(1):35-42.

Hernández, H H.; Benavides-Mendoza, A.; Ortega-Ortiz, H.; Hernández-Fuentes, A. D. and Juárez-Maldonado, A. 2017. Cu Nanoparticles in chitosan-PVA hydrogels as promoters of growth, productivity and fruit quality in tomato. Emirates J. Food Agric. 29(8):573-580.

Hernández-Hernández, H.; González-Morales, S.; Benavides-Mendoza, A.; Ortega-Ortiz, H.; Cadenas-Pliego, G. and Juárez-Maldonado, A. 2018a. Effects of chitosan-PVA and Cu nanoparticles on the growth and antioxidant capacity of tomato under saline stress. Molecules. 23(1):178-192.

Hernández-Hernández, H.; Juárez-Maldonado, A.; Benavides-Mendoza, A.; Ortega-Ortiz, H.; Cadenas-Pliego, G.; Sánchez-Aspeytia, D. and González-Morales, S. 2018b. ChitosanPVA and copper nanoparticles improve growth and overexpress the SOD and JA genes in tomato plants under salt stress. Agronomy. 8(9):175-185.

Kasana, R. C.; Panwar, N. R.; Kaul, R. K. and Kumar, P. 2017. Biosynthesis and effects of copper nanoparticles on plants. Environ. Chem. Letters. 15(2):233-240. 
López-Vargas, E. R.; Ortega-Ortíz, H.; Cadenas-Pliego, G.; de Alba Romenus, K.; Cabrera de la Fuente, M.; Benavides-Mendoza, A. and Juárez-Maldonado, A. 2018. Foliar application of copper nanoparticles increases the fruit quality and the content of bioactive compounds in tomatoes. Appl. Sci. 8(7):1020-1034.

Padayatt, S. J.; Daruwala, R.; Wang, Y.; Eck, P. K.; Song, J.; Koh, W. S. and Levine, M. 2001. Vitamin C: from molecular actions to optimum intake. Handbook of antioxidants. CRC Press. Washington, DC. USA. 117-145.

Pérez-Labrada, F.; López-Vargas, E. R.; Ortega-Ortiz, H.; Cadenas-Pliego, G.; BenavidesMendoza, A. and Juárez-Maldonado, A. 2019. Responses of tomato plants under saline stress to foliar application of copper nanoparticles. Plants. 8(6):151-167.

Pestovsky, Y. S. and Martínez-Antonio, A. 2017. The use of nanoparticles and nanoformulations in agriculture. J. Nanoscie. Nanotechnol. 17(12):8699-8730.

Pinedo-Guerrero, Z. H.; Hernández-Fuentes, A. D.; Ortega-Ortiz, H.; Benavides-Mendoza, A. and Cadenas-Pliego, G. 2017. Cu nanoparticles in hydrogels of chitosan-PVA affects the characteristics of post-harvest and bioactive compounds of jalapeño pepper. Molecules. 22(6):926.

Pradhan, S.; Patra, P.; Mitra, S.; Dey, K. K.; Basu, S.; Chandra, S.; and Goswami, A. 2015. Copper nanoparticle (CuNP) nanochain arrays with a reduced toxicity response: a biophysical and biochemical outlook on vigna radiata. J. Agric. Food Chem. 63(10):2606-2617.

Rajput, V. D.; Minkina, T.; Suskova, S.; Mandzhieva, S.; Tsitsuashvili, V.; Chapligin, V. and Fedorenko, A. 2018. Effects of copper nanoparticles (CuO NPs) on crop plants: a mini review. Bionanoscience. 8(1):36-42.

Ramos, S. J.; Faquin, V.; Guilherme, L. R. G.; Castro, E. M.; Ávila, F. W.; Carvalho, G. S. and Oliveira, C. 2010. Selenium biofortification and antioxidant activity in lettuce plants fed with selenate and selenite. Plant Soil Environ. 56(12):584-588.

Rodríguez, A. T.; Ramírez, M. A.; Falcón, A.; Utria, E. y Bautista, S. 2006. Estimulacion de algunas enzimas en plantas de arroz (Oryza sativa, L.) tratadas con un hidrolizado de quitosana. Cultivos Tropicales. 27(2):87-91.

Rodríguez-Pedroso, A. T.; Ramírez-Arrebato, M. Á.; Cárdenas-Travieso, R. M.; FalcónRodríguez, A. y Bautista-Baños, S. 2006. Efecto de la quitosana en la inducción de la actividad de enzimas relacionadas con la defensa y protección de plántulas de arroz (Oryza sativa L.) contra Pyricularia grisea Sacc. Rev. Mex. Fitopatol. 24(1):1-7.

Rodríguez-Pedroso, A. T.; Ramírez-Arrebato, M. A.; Rivero-González, D.; Bosquez-Molina, E.; Barrera-Necha, L. L. y Bautista-Baños, S. 2009. Propiedades químico-estructurales y actividad biológica de la quitosana en microorganismos fitopatógenos. Rev. Chapingo. Ser. Hortic. 15(3):307-317.

Rouphael, Y. and Colla, G. 2020. Biostimulants in agriculture. Frontiers in Plant Sci. 11(1):40-47. Saharan, V.; Sharma, G.; Yadav, M.; Choudhary, M. K.; Sharma, S. S.; Pal, A. and Biswas, P. 2015. Synthesis and in vitro antifungal efficacy of $\mathrm{Cu}$-chitosan nanoparticles against pathogenic fungi of tomato. Inter. J. Biol. Macromol. 75(1):346-353.

Sánchez, G. R.; Mercado, E. C.; Peña, E. B.; Cruz, H. R. y Pineda, E. G. 2010. El ácido salicílico y su participación en la resistencia a patógenos en plantas. Biológicas. 12(2):90-95.

Santos-Sánchez, N. F.; Salas-Coronado, R.; Villanueva-Cañongo, C. and Hernández-Carlos, B. 2019. Antioxidant compounds and their antioxidant mechanism. London, UK. IntechOpen. $1-28 \mathrm{p}$. 
Sierra-Ávila, R. M. P.; Cadenas-Pliego, G.; Avila-Orta, C.; Betancourt, R.; Jiménez-Regalado, E.; Jiménez-Barrera, R. and Martínez, G. 2014. Synthesis of copper nanoparticles coated with nitrogen ligands. J. Nanomater. 2014(1):74-82.

Sierra-Ávila, R.; Pérez-Alvarez, M.; Cadenas-Pliego, G.; Comparán Padilla, V.; Ávila-Orta, C.; Pérez Camacho, O.; Jiménez-Regalado, E.; Hernández-Hernández, E. and JiménezBarrera, R. M. 2015. Synthesis of copper nanoparticles using mixture of allylamine and polyallylamine. J. Nanomaterials. 361797(1):1-8.

Somasundaran, P.; Fang, X.; Ponnurangam, S. and Li, B. 2010. Nanoparticles: Characteristics, mechanisms and modulation of biotoxicity. KONA powder and particle journal. 28(1):38-49.

Steiner, A. A. 1961. A universal method for preparing nutrient solutions of a certain desired composition. Plant Soil. 15(2):134-154.

Sykłowska-Baranek, K.; Pietrosiuk, A.; Naliwajski, M. R.; Kawiak, A.; Jeziorek, M.; Wyderska, S. and Chinou, I. 2012. Effect of 1-phenylalanine on PAL activity and production of naphthoquinone pigments in suspension cultures of Arnebia euchroma (Royle) Johnst. In vitro Cellular \& Developmental Biology-Plant. 48(5):555-564.

Usman, M.; Farooq, M.; Wakeel, A.; Nawaz, A.; Cheema, S. A.; ur Rehman, H. and Sanaullah, M. 2020. Nanotechnology in agriculture: Current status, challenges and future opportunities. Sci. Total Environ. 721(1):137778.

Yang, W.; Fortunati, E.; Bertoglio, F.; Owczarek, J. S.; Bruni, G.; Kozanecki, M. and Puglia, D. 2018. Polyvinyl alcohol/chitosan hydrogels with enhanced antioxidant and antibacterial properties induced by lignin nanoparticles. Carbohydrate Polymers. 181(1):275-284.

Yang, W.; Owczarek, J. S.; Fortunati, E.; Kozanecki, M.; Mazzaglia, A.; Balestra, G. M. adn Puglia, D. 2016. Antioxidant and antibacterial lignin nanoparticles in polyvinyl alcohol/chitosan films for active packaging. Industrial Crops and Products. 94(1):800-811.

$\mathrm{Yu}, \mathrm{Z}$. and Dahlgren, R. A. 2000. Evaluation of methods for measuring polyphenols in conifer foliage. J. Chem. Ecol. 26(9):2119-2140.

Zhang, X. and Liu, C. J. 2015. Multifaceted regulations of gateway enzyme phenylalanine ammonia-lyase in the biosynthesis of phenylpropanoids. Mol. Plant. 8(1):17-27. 